\title{
Spatial Correlation of the Backscattered Ultrasonic Grain Noise in the Ultrasonic Inspection of the Forging Titanium Alloy
}

\author{
Theodor Tranca ${ }^{1}$, Iuliana Radu ${ }^{2}$ \\ ${ }^{1}$ Diac Service Srl, Bucharest, Romania \\ ${ }^{2}$ Zirom SA, Giurgiu, Romania
}

Email address:

teodortranca@yahoo.com (T. Tranca)

\section{To cite this article:}

Theodor Tranca, Iuliana Radu. Spatial Correlation of the Backscattered Ultrasonic Grain Noise in the Ultrasonic Inspection of the Forging Titanium Alloy. International Journal of Mineral Processing and Extractive Metallurgy. Vol. 6, No. 3, 2021, pp. 33-40.

doi: 10.11648/j.ijmpem.20210603.11

Received: June 14, 2021; Accepted: July 5, 2021; Published: July 15, 2021

\begin{abstract}
Ultrasonic inspection is a routine Non Destructive Examination (NDE) method adopted by the aircraft engine manufacturers. However, the detection of smaller defects in such materials is made difficult by the complicated ultrasoundmicrostructure interactions. One of the adverse influences of the interactions is the high backscattered grain noise level accompanying with the ultrasonic inspections of some titanium alloys. The high grain noise deteriorates the Signal to Noise ratio $(\mathrm{S} / \mathrm{N})$ of pulse/echo inspections and consequently may lead to the missing detection of an existing flaw. Ultrasonic signal fluctuations have direct impact on flaw detection, flaw characterization and the estimation of the Probability of Detection (POD). The total backscattering is controlled by grain morphology, grain orientation and elastic anisotropy, which may vary throughout the microstructure. Thus any Thermo-Mechanical Processing (TMP) leading to the variations of material microstructure may influence the backscattered grain noise. We developed new ideas of how to extract useful microstructural information from the forging simulation software Simufact.forming, a commercial software package produced by Simufact Engineering $G m b H$. A model is then developed to correlate the grain noise signals with the microstructural variations due to the inhomogeneous plastic deformation associated with the forging processing. The grain noise levels predicted by the model at various locations are compared with experiments. Reasonably good agreements are observed.
\end{abstract}

Keywords: Ultrasonic Grain Noise, Simulation Software, Elastic Anisotropy

\section{Introduction}

In principle, the noise caused by the structure of polycrystalline materials is the result of the inhomogeneity of the acoustic properties of the material. These inhomogeneities are primarily associated with the anisotropy of the elasticity constants in grains or crystals. Total scattered radiation can be controlled by grain morphology, grain orientation, and elastic anisotropy of the microstructure.

Large variations in material noise were observed in connection with the position and direction of examination for Ti-6Al-4V slabs. Such variations are supposed to be correlated with variations of the microstructure that come from the thermo-mechanical process.

The thermo-mechanical process is the most used way to control the microstructure and consequently the mechanical properties (for example imposing restrictions on the working temperature and the degree of deformation). A large variety of microstructural characteristics of the final product (grain size, degree of recrystallization, texture, etc.), [1] are related to the parameters of thermo-mechanical processes.

Thus, in order to be able to anticipate the ultrasonic behavior of a material, it is essential to anticipate its structural characteristics as a result of TMP, for which purpose we use computerized prediction techniques of the forging process. [2]

\section{Material Noise Prediction Elements}

A typical thermo-mechanical process is the one applied in 
the case of forging ingots (Figure 1). The cast ingot is transformed into a cylindrical billet by reducing the diameter and then by forging it is brought to a shape as close as possible to the final product.

The microstructure of the ticket will depend on the microstructure of the casting and the history of the thermomechanical process. [3] In the cast ingot, the grains are typically equiaxi, but due to the drastic reduction in diameter, the typical microstructure of the billet will be with elongated macro-grains (primary $\beta$ ), consisting of $\alpha$ colonies of hexagonal crystals created by solid state transformations with the orientation determined in the piece of that of the initial $\beta$ grains. The final microstructure of the forging is dependent on the specifics of the forging process (forging temperature, processing time, deformation rate, cooling rate and subsequent heat treatment). [4]

Apart from the morphology of the structure, the inhomogeneities of the plastic deformations during forging induce a preferential orientation of the crystals, a texture formed by the cleavage or maclation of the crystals, or a combination between the two. [5]

Such deformations on which the texture depends will act as the elastic anisotropy factor $\left\langle\delta \mathrm{C}_{\mathrm{ij}} \delta \mathrm{C}_{\mathrm{kl}}\right\rangle$ vary with the position and direction inside the slab. These variations influence the material noise.

Due to these deformations, the deformation texture appears, which is conventionally represented by polar figures that describe the statistical distribution of the hexagonal basal plane. The extent and intensity of the texture are dependent on the volume of deformation and the temperature at which it was performed. [6, 7]

Due to the influence of inhomogeneous plastic deformation, the microstructure is expected to have local variations in grain morphology, grain orientation and texture.

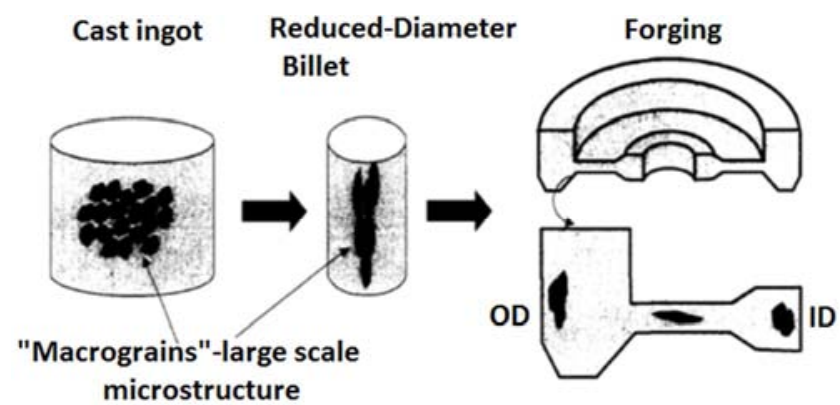

Figure 1. Typical forging process accompanied by deformation and orientation of the grain depending on their position in the final product. [1]

However, for large slabs it is difficult to give photographic details of structural variations only through traditional metallographic approaches. [8] Numerical simulations were carried out in order to do a comparison between numerical data and experimental results. The simulations were carried out using an implicit commercial code able to conduct coupled thermo-mechanical-microstructural analysis of hot forming processes of metal alloys. [9]

For this purpose there are specialized software packages such as DEFORM® or Simufact.forming. An example of using these programs is given in Figure 2 where the case of a forged cylindrical ticket type is presented. The figure shows the "stress map" where the variations of the plastic deformation stresses are highlighted according to their position in the part, in a section of the forged billet.

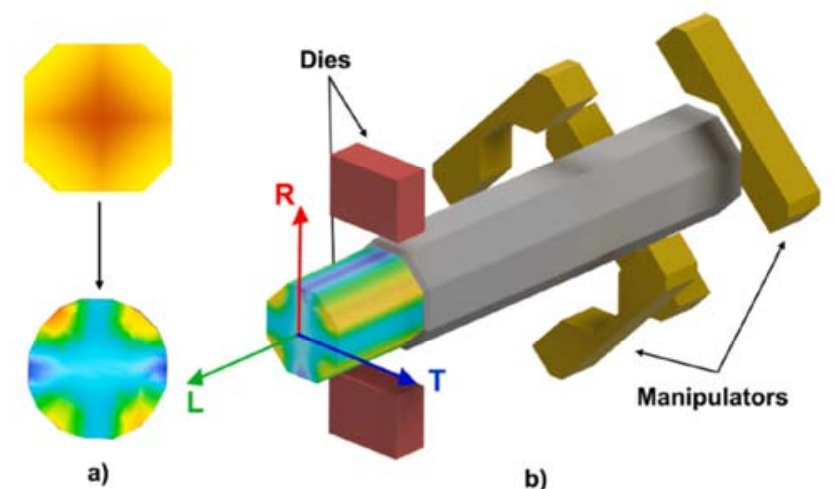

Figure 2. (a) Initial VAR ingot square cross section with no strain induced top and DEFORM ${ }^{\circledR}$ simulation of the cross-section strain induced during the primary forging, (bottom) [orange/yellow coloring relates to total high strain regions]. (b) $3 D$ schematic of a typical open die forge / titanium alloy work piece interaction during the primary forging process with strain induced overlaid. [10]

An approach was thus developed through a mathematical model that can be predictable from the noise of material deduced from variations in microstructure, which in turn are dependent on plastic deformations during the forging process. It is possible to anticipate and thus determine the noise of the material and implicitly the quality of the material from the point of view of the ultrasonic control, in different areas of a forged part, by modelling the forging technology. [11]

For this purpose, we start from the hypothesis of the elements that cause the scattering of ultrasonic radiation in the bill, having a specific orientation and elongation. These elements are a consequence of the forging process that changes their shape and orientation and introduces the deformation texture. The position and texture of these elements in the slab can be deduced by applying the program Simufact.forming produced by Simufact Engineering $\mathrm{GmbH}$.

\subsection{The Relationship between the Elastic Constants of Two Points Located in a Textured Biphasic Microstructure}

To predict an absolute noise levels we need detailed knowledge of the metal microstructure which enters the model calculations through certain frequency-dependent factors known as "backscatter coefficients" or "Figures-ofMerit" (FOM). The resulting expression for "noise" produced under these conditions contains a factor which depends on the volumetric density of scatters and their Root Mean Square (RMS) scattering amplitude in the backscattered direction. This term, which is called FOM, appears to be a material property useful in characterizing microstructure. 
Under the assumption that the solid density is independent of position, FOM $(\omega)$ and $\eta(\omega)$ for longitudinal waves propagating in the in $\mathrm{S} 3$ direction are related to microstructure features by eqn. (1):

$$
\operatorname{FOM}(\omega)^{2}=\eta(\omega)=\mathrm{K} /\left(4 \pi \rho \mathrm{Vt}^{2}\right)^{2} \int d^{3} \vec{S}\left\langle\delta \mathrm{C}_{33}(\vec{r}) \delta \mathrm{C}_{33}\left(\overrightarrow{r^{\prime}}\right)\right\rangle e^{2 i k S 3}(1)
$$

where:

$\eta(\omega)$ - backscattering power coefficient

$\omega$ - is the angular frequency,

$\mathrm{k}$ - is the magnitude of the wave vector of the incident wave,

$\rho$ - is the density of the solid,

$\mathrm{Vt}$ - is the longitudinal wave velocity,

$\mathrm{S}=\vec{r}-\overrightarrow{r^{\prime}}$ - is a vector defined by the two points $\vec{r}$ and $\overrightarrow{r^{\prime}}$ in the solid medium,

$\mathrm{S}_{3}$ - is the component of $\vec{S}$ in the direction of wave propagation (3-direction),

$\delta \mathrm{C}_{33}(\vec{r})$ - is the local deviation of the elastic constant from its Voigt average,

$\delta \mathrm{C}_{33}\left(\mathrm{r}^{\rightarrow}\right)=\left(\mathrm{C}_{33}-\mathrm{C}_{33}{ }^{\text {Voigt }}\right)$, and the notation 〈...〉 denotes an ensemble average,

$\left\langle\delta \mathrm{C}_{33}\left(\mathrm{r}^{-}\right) \delta \mathrm{C}_{33}\left(\mathrm{r}^{\prime}\right)\right\rangle$ is known as the two-point correlation of elastic constants, which describes the correlation in the perturbation in the elastic stifnesses.

For single-phase materials which are macroscopically homogeneous and have a random microstructure orientation, $<\mathrm{C}_{33}\left(\mathrm{r}^{\rightarrow}\right) \delta \mathrm{C}_{33}\left(\mathrm{r}^{\prime \rightarrow}\right)>$ may be written in the form:

$$
<\delta \mathrm{C}_{33}\left(\mathrm{r}^{\rightarrow}\right) \delta \mathrm{C}_{33}\left(\mathrm{r}^{\prime}\right)>=<\delta \mathrm{C}_{33} \delta \mathrm{C}_{33}>\mathrm{W}\left(\mathrm{r}^{\overrightarrow{ }}-\mathrm{r}^{\prime \rightarrow}\right)
$$

where:

$<\delta \mathrm{C} 33 \delta \mathrm{C} 33>-$ is a constant controlled by the crystal anisotropy;

$\mathrm{W}\left(\mathrm{r} \mathrm{r}^{-}-\mathrm{r}^{\prime}\right)$ - the probability that two points separated by the distance $\left(\mathrm{r} \mathrm{r}^{-}-\mathrm{r}^{\prime \prime}\right)$ in the solid will be in the same crystal;

$<\delta \mathrm{C} 33 \delta \mathrm{C} 33>$ can be considered as an average of the solid angle (C33 - C33Voigt) for a single crystal. [12]

$$
\operatorname{FOM}(\omega)^{2}=\eta(\omega)=\left(\frac{\omega^{2}}{4 \pi \rho V_{l}^{2}}\right)^{2}<\delta C_{33} \delta C_{33}>\int d^{3} \vec{S} \mathrm{~W}(\vec{S}) e^{2 i k S_{3}}
$$

For the ellipsoidal element causing scattered radiation shown in Figure 3, which is assumed to be axially symmetrical along the $Z$ axis and the semi-axes $a_{m}$ and $c_{m}$, eqn. (3) can be written as in eqns. $(4 \div 7)$.

$$
\operatorname{FOM}(\omega)^{2}=\eta(\omega)=\left(\frac{\omega^{2}}{4 \pi \rho V_{l}^{2}}\right)^{2}<\delta C_{33} \delta C_{33}>\int_{0}^{2 \pi} \int_{0}^{\pi} \frac{2 \sin \theta}{A^{3}} d \theta d \Phi
$$

$\mathrm{A}=\frac{\left\{1+\left(R^{2}-1\right) \cos \theta^{2}\right\}^{2}}{(3 V R / 4 \pi)^{1 / 3}}-2 \mathrm{ik}(\sin \theta \sin \Phi \sin \tau+\cos \theta \cos \tau)$ (5)

$\mathrm{R}=\frac{a_{m}}{c_{m}}-$ the ratio of the dimensions of the element causing the scattering

$\mathrm{V}=\frac{4 \pi a_{m}^{3}}{3 R}-$ the average volume of the element causing the scattering

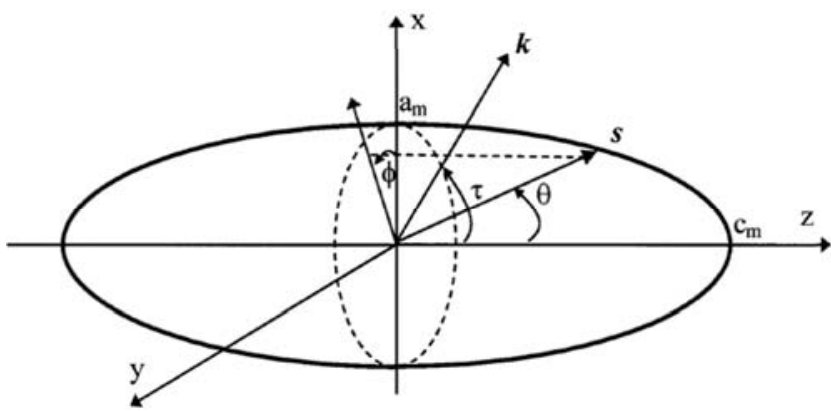

Figure 3. Geometry of an ellipsoidal scattering element, sonic beam propagation is assumed to be parallel to vector $k$. [1]

When the variations and intensity of the texture are small, its effect in correlating the elasticity functions between two points is given by the term $<\delta \mathrm{C} 33 \delta \mathrm{C} 33>$. Due to the presence of texture, this term may change depending on the direction of examination.

The intensity and type of texture are defined as functions of orientation of the crystals, respectively by the probability that a certain crystal has a specific orientation in relation to the coordinate axes of the respective sample.

Specifically, the representation of a crystal in the microstructure is made by a set of Euler angles $\theta, \psi, \phi$. The orientation function of the crystals is represented by $\mathrm{w}(\xi, \psi$, $\phi)$, where

$\xi=\cos \theta$ and $w(\cos \theta, \psi, \phi) \sin \theta \mathrm{d} \theta \mathrm{d} \psi \mathrm{d} \phi$ is the probability of finding crystals oriented in the angular domain $\mathrm{d} \theta \mathrm{d} \psi \mathrm{d} \phi .[13]$

Obviously,

$$
\int_{0}^{2 \pi} \int_{0}^{2 \pi} \int_{-1}^{1} w(\xi, \psi, \phi) d \xi d \psi d \phi=1
$$

Once w $(\xi, \psi, \phi)$ is defined, the effect of the texture can be evaluated. In our case,

$<\delta \mathrm{C} 33 \delta \mathrm{C} 33>$ with texture can be evaluated with the eqn. (9). [2]

$$
\begin{aligned}
& \delta \mathrm{C}_{33} \delta \mathrm{C}_{33}>= \\
& \int_{0}^{2 \pi} \int_{0}^{2 \pi} \int_{-1}^{1}[\delta \mathrm{C} 33(\xi, \psi, \phi) \delta \mathrm{C} 33(\xi, \psi, \phi)] w(\xi, \psi, \phi) d \xi d \psi d \phi(9)
\end{aligned}
$$

The term $\delta \mathrm{C} 33(\xi, \psi, \phi)$ in eqn. (9) represents the local deviation from Voigt's average of the homogeneous alpha phase macrostructure, respectively $\delta \mathrm{C} 33(\xi, \psi, \phi)=\mathrm{C} 33(\xi, \psi$, $\phi)-\mathrm{C} 33$ Voigt. Consider the basal plane of the crystals normal to the flow direction of the metal during plastic deformation, as shown in Figure 4.

Mathematically, the function $\mathrm{w}(\xi, \psi, \phi)$ is a function only of $\xi$ or $\theta(\xi=\cos \theta)$. It is not possible to assess exactly to what extent the distribution of the basal poles changes with respect to $\theta$. For reasons of mathematical correctness, $w(\xi)$ is expressed in the Gaussian form $w(\xi)=\mathrm{C} e^{-(\xi / \Delta)^{2}}$. By the normalization conditions established in eqn. (8), the constant $\mathrm{C}$ is defined in eqn. (10), where erf represents the function error. [13]

$$
w(\xi))=\frac{1}{\left[4 \pi^{5 / 2} \Delta \operatorname{erf}\left(\frac{1}{\Delta}\right)\right]} e^{-(\xi / \Delta)^{2}}
$$


Z: Normal of the metal flow direction

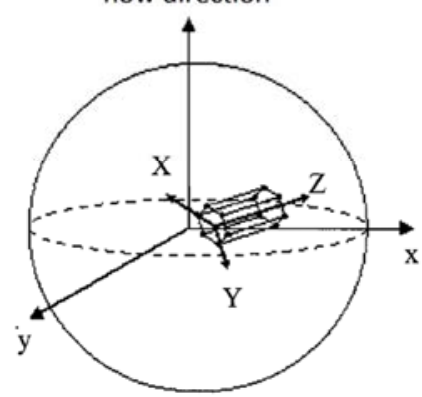

Figure 4. Crystallite alignment within forging. [2]

The term $\Delta$ is the one that determines the intensity of the texture. When $\Delta$ becomes very large, the texture disappears so that $\mathrm{W}(\xi)$ is a constant for a randomly distributed environment.

The small values of $\Delta$ correspond to a very pronounced texture. It is known that the intensity of the texture is determined by the volume of labor performed on the material or by the internal stresses resulting from its plastic deformations.

The relation between $\Delta$ and the internal tensions $\varepsilon_{\mathrm{e}}$ is given by eqn. (11).

$$
\Delta=\frac{P 1}{\sqrt{\varepsilon_{\mathrm{e}}}}
$$

Where $P 1$ is an adjustment parameter that can be deduced by comparing theoretical predictions with a number of experiments. Finally, the function $w(\xi)$ can be expressed relative to the internal stresses $\varepsilon_{e}$ according to eqn. (12).

$$
\mathrm{W}(\xi)=\frac{1}{\left\lfloor 4 \pi^{5 / 2} \operatorname{P} 1 \operatorname{erf}\left(\frac{\sqrt{\varepsilon \mathrm{e}}}{P 1}\right)\right\rfloor} e^{-\xi^{2} \varepsilon \mathrm{e} / P 1^{2}}
$$

The effect of the texture $<\delta \mathrm{C} 33 \delta \mathrm{C} 33>$ can be evaluated by replacing $\mathrm{w}(\xi)$ in eqn. (9) with the expression from eqn. (12). A specific example is given by Figure 5 and 6 in which a simulation performed using the program Simufact.forming 12.0., simulation program in the field of forging technologies is presented.

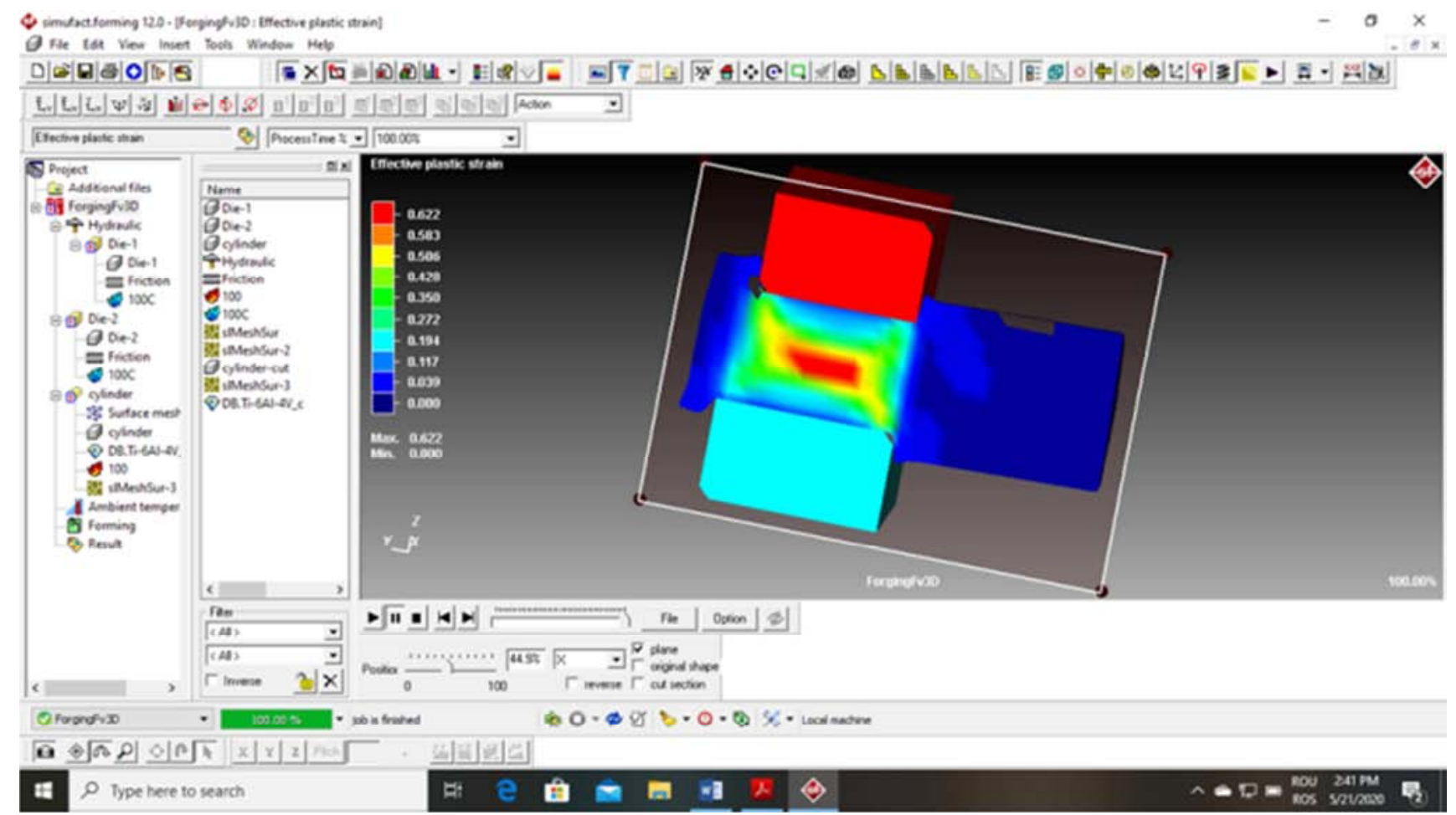

Figure 5. Plastic deformation stress map.

In this case, we started from a Ti6Al-4V ingot with a diameter of $400 \mathrm{~mm}$ and forged to a final diameter of 250 $\mathrm{mm}$. Both the map of internal stresses and that of the material flow directions clearly indicate that these directions are rigorously aligned with the radial forging directions. As shown in Figure 6, the basal plane of the crystals tends to align with the direction of flow of the metal. Since the values of the internal stresses can be evaluated with the help of the stress map obtained with the help of the Simufact.forming program, (Figure 5) the exact value of $<\delta \mathrm{C} 33 \delta \mathrm{C} 33>$ can be determined with eqn. (12) and eqn. (9) if $P 1$ is known. For biphasic structures $(\alpha+\beta)$, the element $<\delta \mathrm{C}_{\mathrm{ij}} \delta \mathrm{C}_{\mathrm{kl}}>$ is smaller than that for pure $\alpha$ structures. To quantify this effect, a second adjustment parameter $P 2$ is entered.

$$
<\delta \mathrm{C}_{\mathrm{ij}} \delta \mathrm{C}_{\mathrm{kl}}>_{\alpha+\beta}=\mathrm{P}_{2}<\delta \mathrm{C}_{\mathrm{ij}} \delta \mathrm{C}_{\mathrm{kl}}>_{\alpha}
$$

\subsection{Calculation of the Coefficient of Power of the Scattered Radiation}

We consider the model parameter $V$ (eqn. 7) constant in the forging volume while the local parameter $<\delta \mathrm{C} 33 \delta \mathrm{C} 33>_{\alpha+\beta}$ is determined by the two adjustment parameters $P 1$ and $P 2$ 
and by the plastic deformation stresses (eqns. $9 \div 13$ ). The calculation of the FOM is made for the direction of radial examination for tickets and normal to the major surface for forged plates. The values of the internal stresses $\varepsilon_{\mathrm{e}}$ (Figure 5) and the orientation angles of the crystals in relation to the angle of incidence of the incident beam (Figure 6) can be determined using the simulation program Simufact.forming, for a specific forging technology, at each point of the examined piece. The FOM calculation is done in several stages, as follows:

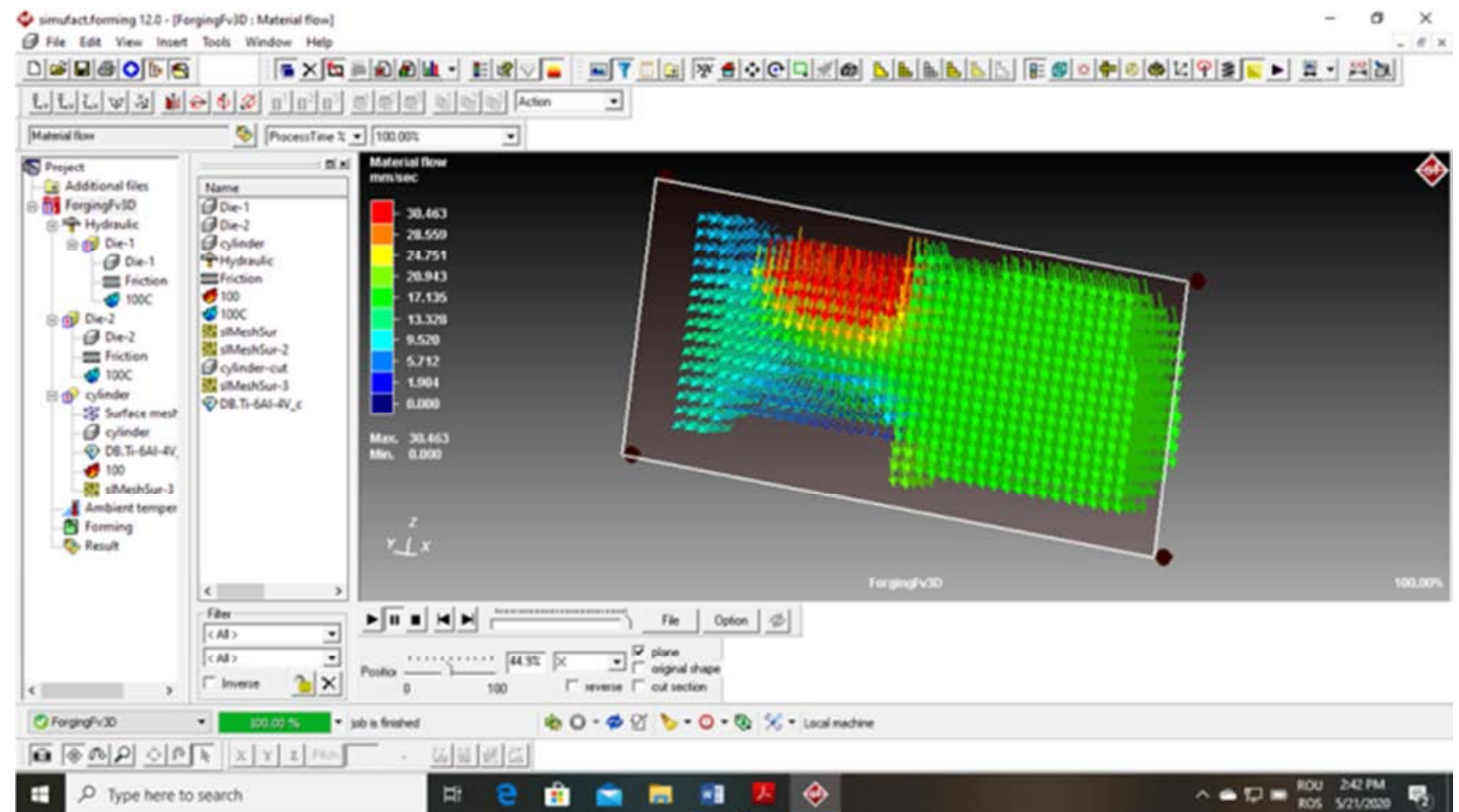

Figure 6. Map of speeds and flow directions of the material.

Stage 1- A FOM-Frequency Dependence Curve is experimentally raised using the stepped reference block provided with Flat Bottom Holes (FBH) 3/64 and 5/64, positioned at different depths (Figure 7). To do this, successively scan the FBH 3/64 at a depth of $129.5 \mathrm{~mm}$ from the inlet surface, with three immersion transducers having the same dimension of the piezoelectric element but different central frequencies (respectively $2.25 \mathrm{MHz}, 5 \mathrm{MHz}$ and 10 $\mathrm{MHz}$ ) and the oscillograms of each scan are recorded (Figures 8, 9 and 10).

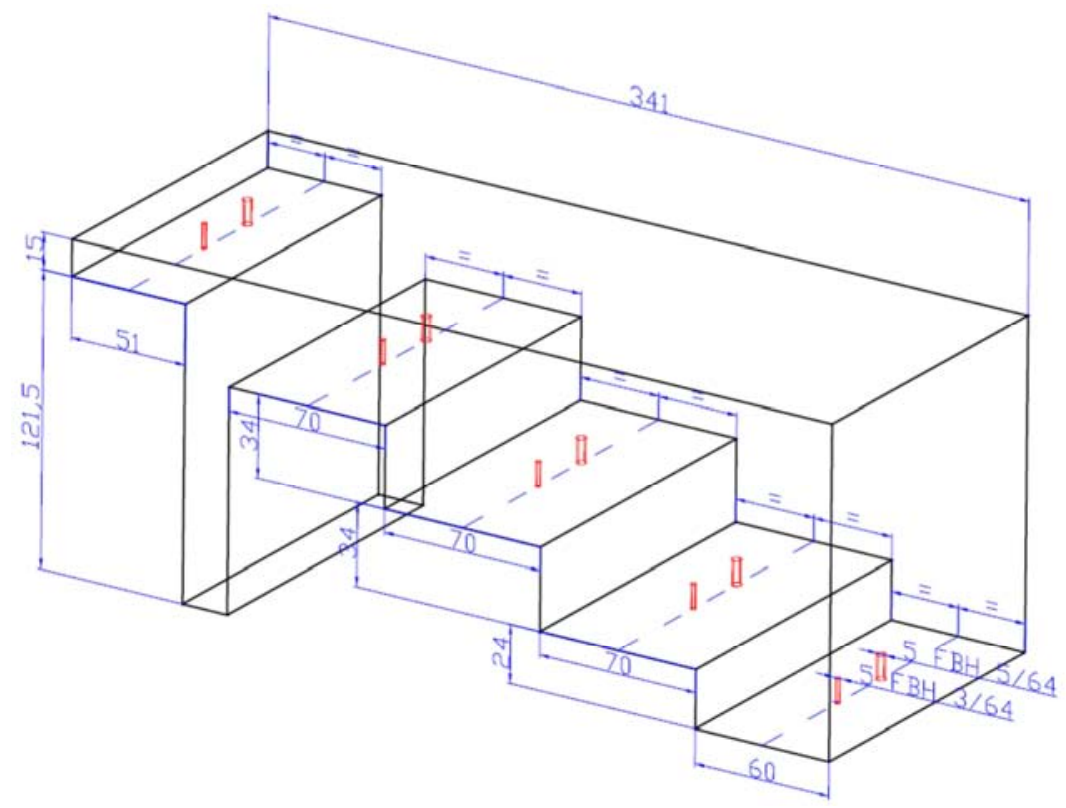

Figure 7. Forged plate examination reference block. 


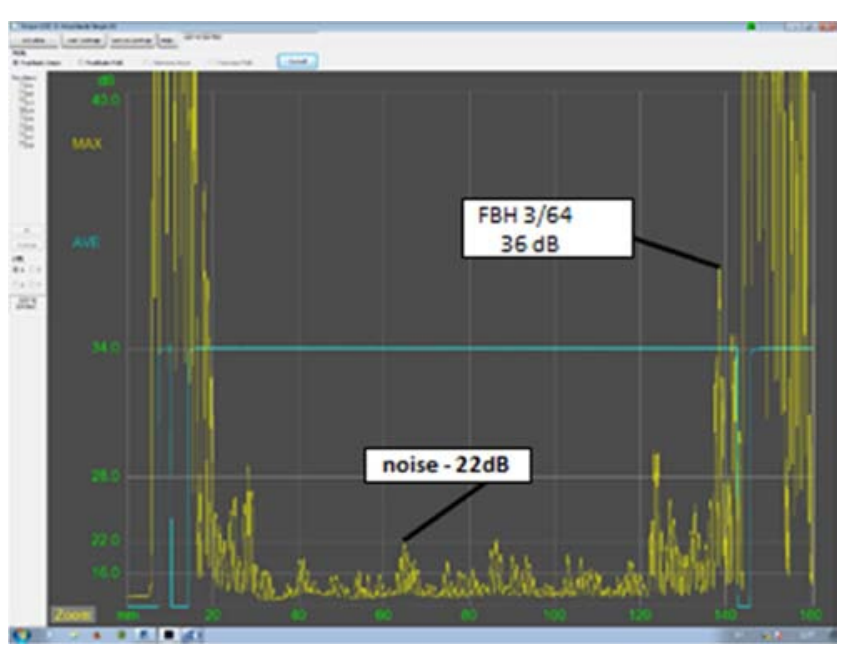

Figure 8. Transducer $\Phi 19 \mathrm{~mm}, 2.25 \mathrm{MHz}$.

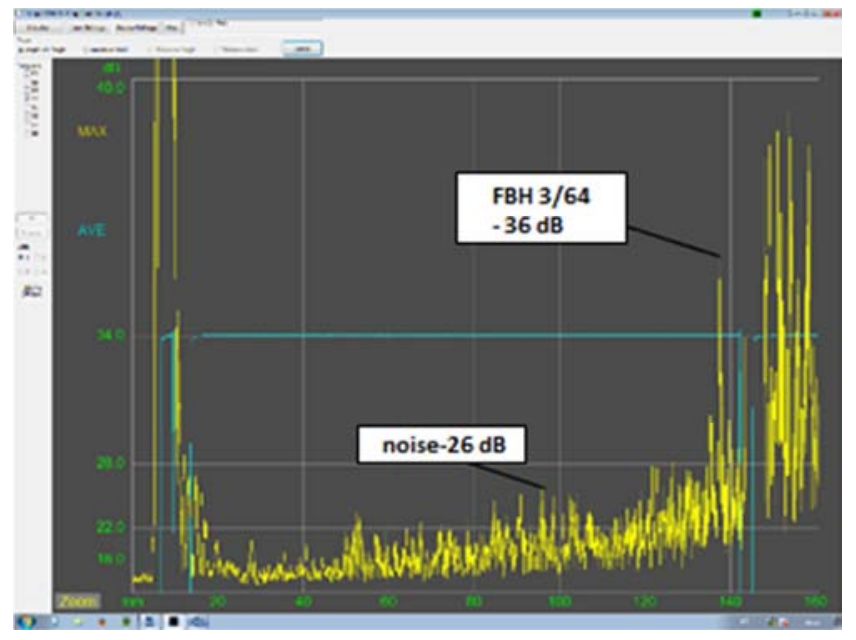

Figure 9. Transducer $\Phi 19 \mathrm{~mm}, 5 \mathrm{MHz}$.

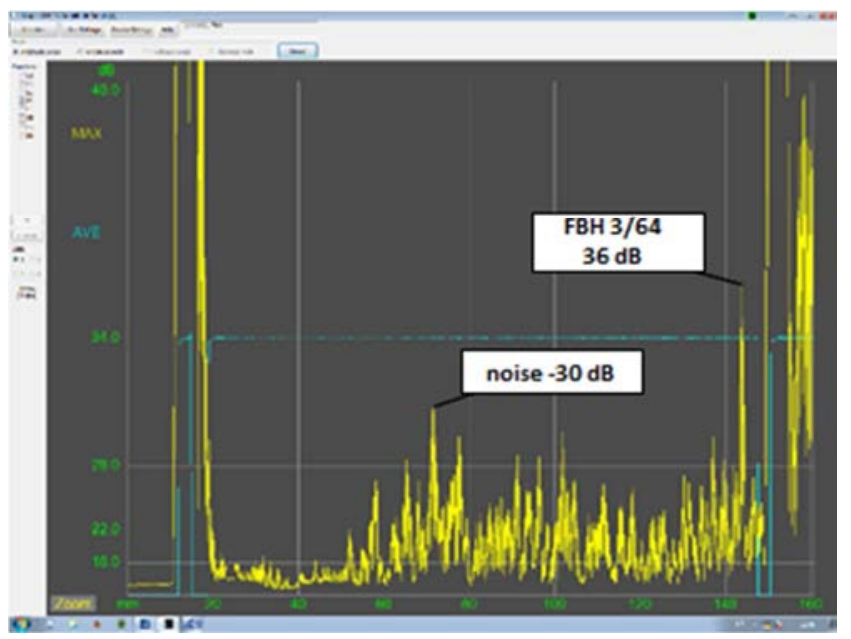

Figure 10. Transducer $\Phi 19 \mathrm{~mm}, 10 \mathrm{MHz}$.

The examination sensitivity will be set so that the reference reflector is at the same relative amplitude, respectively FBH $3 / 64$ echo at $60 \%$ of Full Screen Height (FSH) for each scan. The material noise level (which is directly proportional to the FOM) will be recorded for each transducer and the $S / N$ ratio for each transducer will be determined. Experimentally, these value of the $S / N$ ratio are determined as follow:

2.25 MHz Transducer- $\mathrm{S} / \mathrm{N}=6: 1$

5.0 MHz Transducer- $\mathrm{S} / \mathrm{N}=4: 1$

10.0 MHz Transducer $-\mathrm{S} / \mathrm{N}=2: 1$

The Signal / Noise ratio can be expressed as a function of the pulse volume and the power coefficient of the scattered radiation $\eta(\omega)$ according to eqn. (14). [14]

$$
\mathrm{S} / \mathrm{N}=\left[\frac{\operatorname{Aflaw}(\omega)}{\sqrt{\eta(\omega)}}\right] \frac{1}{\sqrt{B^{2} \Delta t p}}
$$

where:

B - average ultrasonic pulse diameter,

$\Delta$ tp- ultrasonic pulse length,

Aflaw- amplitude of the reference defect at $60 \% \mathrm{FSH}$.

To determine the pulse volume, a FBH 3/64 located at a depth of $129.5 \mathrm{~mm}$ is used for the pulse diameter and the bottom echo from the lower surface of the step of $136 \mathrm{~mm}$ (Figure 7) to determine the pulse length. [15]

Given these data and the amplitude of the reference defect Aflaw, the same $(60 \% \mathrm{FSH})$ for all examinations, we can determine the conversion factor between FOM and $\mathrm{S} / \mathrm{N}$ ratio for each transducer, respectively each examination frequency. The dependency graph between FOM and frequency can now be drawn (Figure 11).

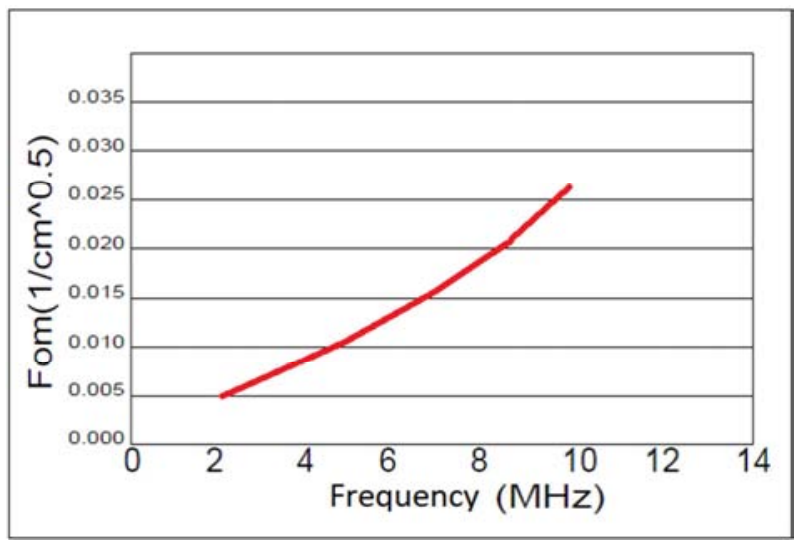

Figure 11. Dependency FOM-Frequency.

Stage 2- The Simufact.forming program will be run and the degree of deformation of the material, volume $V$ and ratio $R$ will be set as initial values in order to obtain the values of the plastic deformation stresses $\varepsilon$ e for the scanned reference block.

Stage 3- An FOM value will be appreciated from the graph in Figure 11 for the frequency with which the examination is performed. Using the eqns. $(3 \div 7)$, from this value determined graphically we can deduce the value $<\delta \mathrm{C} 33 \delta \mathrm{C} 33>$ from which we can later deduce the value $\Delta$ from eqn. (11) and knowing the value $\varepsilon \mathrm{e}$ deduced in Stage 2, the value of the adjustment parameter $P 1$ can be deduced.

Stage 4 - Having the value of the adjustment parameter $P 1$, and with the help of the simulation program Simufact. forming from which the angle of inclination of the basal plane is extracted in relation to the incident beam $\tau$, volume 
$V$ and ratio $R$, we can calculate FOM (eqn. 4) for any forged part like the one in Figure 12, at any point thereof. Since the FOM value is also related to the $\mathrm{S} / \mathrm{N}$ ratio, for the same transducer and on approximately equal working fields, it can be evaluated by comparing the FOM values obtained by calculation on scan areas 1, 2 and 3 (Figure 12) with the value obtained on the reference block, which from the areas of the plate to be examined meets the $\mathrm{S} / \mathrm{N}$ ratio conditions required by the standard.
Another element of material noise prediction is the orientation of the crystals according to the scanning direction. This orientation is related to the direction of flow of the material.

(Figure 13) and can be anticipated in order to determine the defining angles for the element causing the scattering (Figure 3), using the same simulation program. The forging technology will be modified accordingly in order to achieve those material and texture conditions that allow the efficient ultrasonic examination of the semi-finished product.

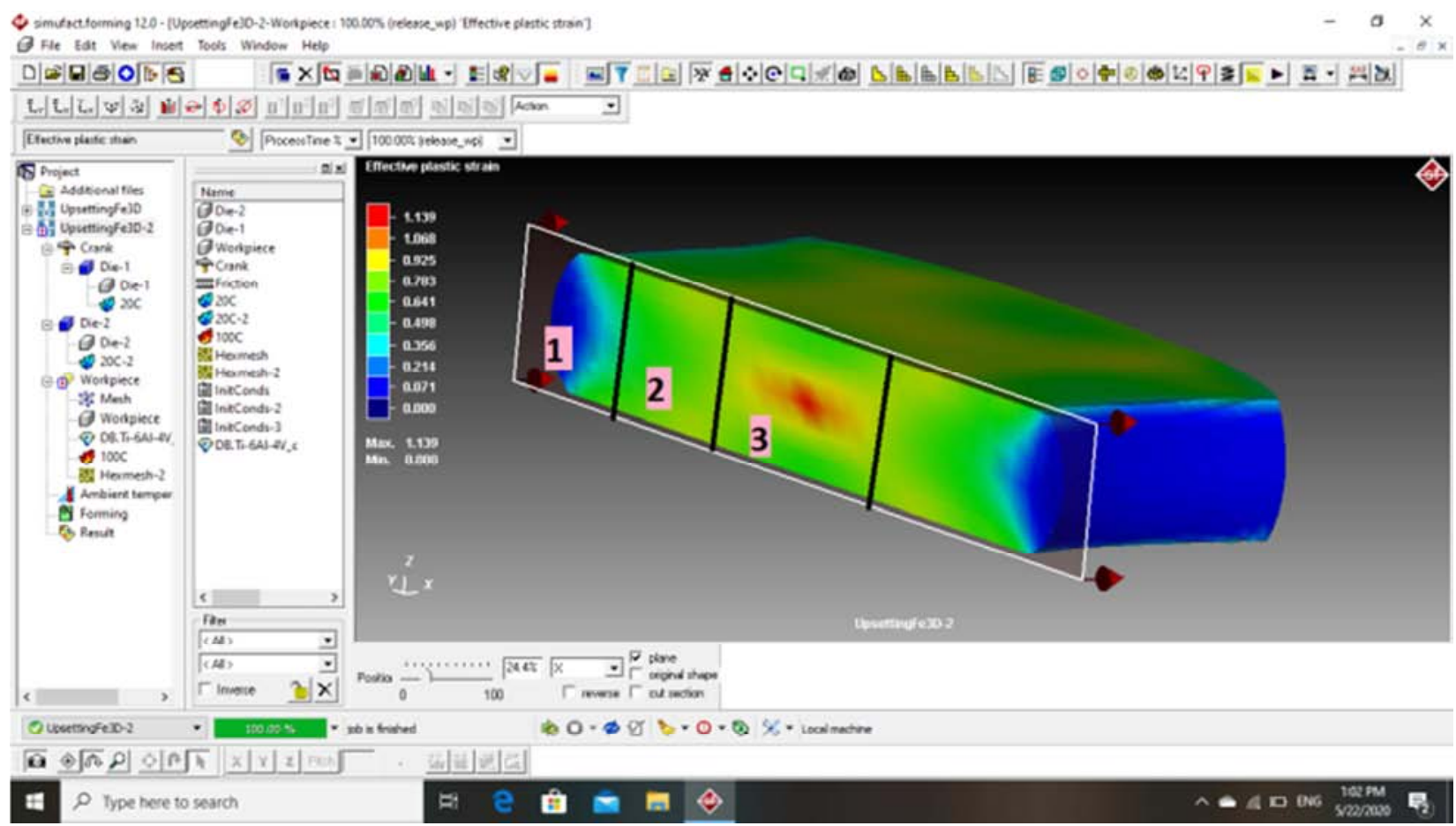

Figure 12. Map of plastic deformation stresses in a forged plate.

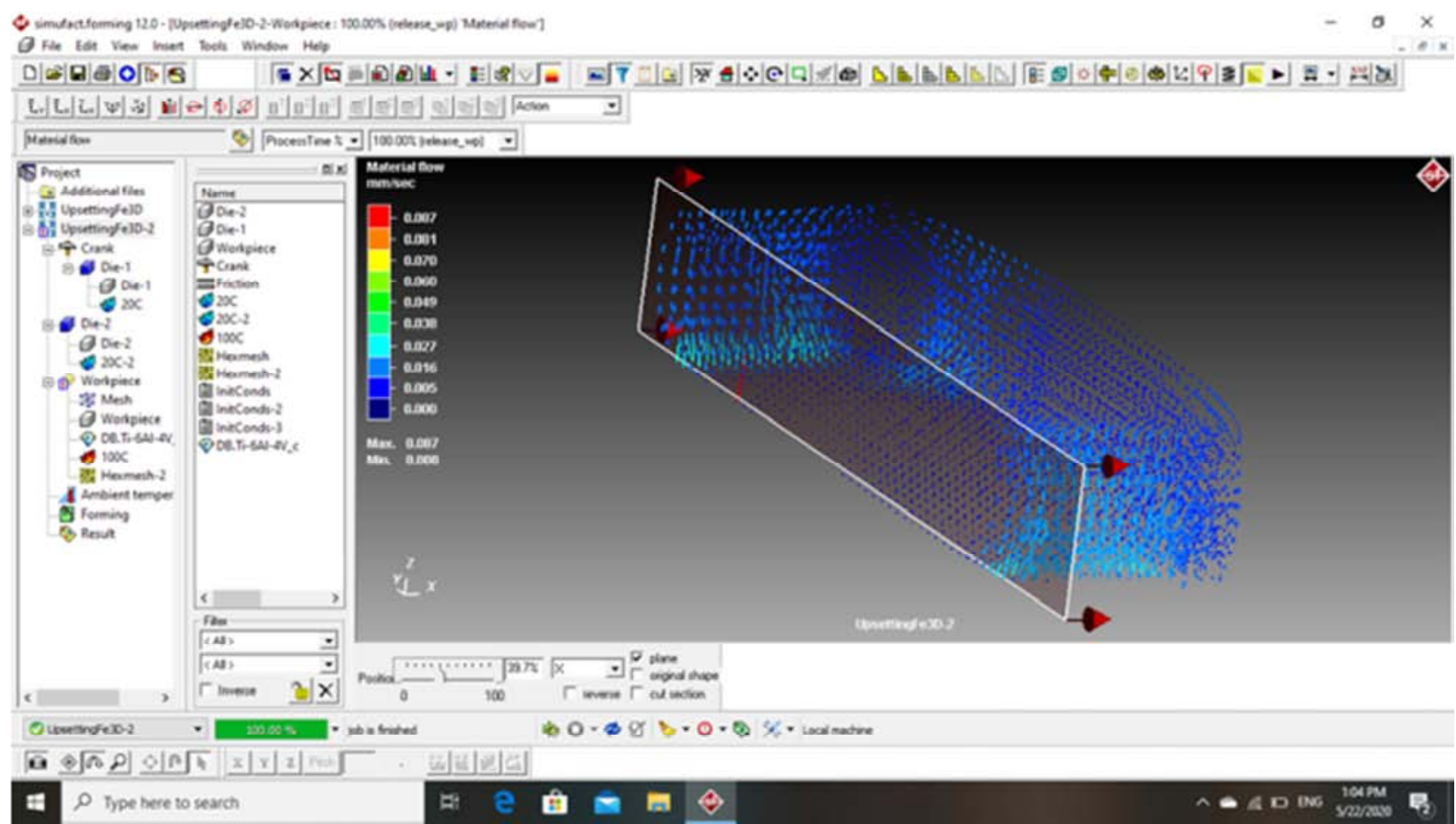

Figure 13. Map of velocities and flow directions of the material in a forged plate. 


\section{Conclusions}

We set out to develop a model that correlates the material noise with the microstructural variations resulting from the plastic deformations during forging. The inputs of this model refer to the average volume $V$ of the element that causes the scattering, supposed to be constant during forging, to the geometric characteristics of this element and to the local texture due to the inhomogeneity of the plastic deformation stresses.

The shape and orientation of the elements causing the scattering can be deduced with the Simufact.forming simulation program and vary over the entire volume of the slab. The other parameters necessary for modeling, respectively the adjustment parameters $P 1$ and $P 2$ for the characterization of the anisotropy of the elastic properties were obtained experimentally.

The aim of this paper is a better understanding of the fundamental ultrasonic properties of Ti-6A1-4V slabs as well as the improvement of inspection and manufacturing procedures of this material.

\section{Acknowledgements}

This work was supported by S. C. ZIROM-S. A - Giurgiu - ROMANIA and was performed by the NDT Consulting Company - DIAC SERVICII srl and AROEND -Romania.

\section{References}

[1] EN 3114-002, Aerospace series-Test method- Microstructure of $(\alpha+\beta)$ titanium alloy wrought products- Part 002: Microstructure of bars, sections, forging stock and forgings.

[2] Ricardo Alves de Sousa and Robertt Valente - Numerical prediction of Biphasic Titanium Alloys Microstructure in Hot Forging Operations, 14th international conference of Metal Forming, (20120).

[3] Aloman A. - The structure, properties and phase equilibrium diagrams of titanium and others of titanium. Bucharest, Polytechnic University, 1996, 274 p.
[4] J Sieniawski, W. Ziaja, K. Kubiak and M. MotykaMicrostructure and Mechanical Properties of High Strength Two-Phase Titanium Alloys, Rzeszów University of Technology, Dept. of Materials Science, Poland- october 2013.

[5] Suciu Valeria, Suciu Marcel Valeriu- Study of Materials FAIR PARTNERS Bucharest, 2008, 255 p.

[6] Yoshinori Ito, Hiroyuki Takamatsu, Shogo Saeki, - Influence of micro-texture on ultrasonic reflection in Ti-6Al-4V alloy hot-forged in $\alpha+\beta$ region -The 14th World Conference on Titanium, 2020.

[7] Y. Ito, H. Takamatsu, and K. Kinoshita: Proceedings of the 13th World Conference on Titanium, 2016, pp. 885-888.

[8] K. Kulcsár et al. Structural analysis of titanium alloys: - 2018 IOP Conf. Ser.: Mater. Sci. Eng. 426012029.

[9] A. Astarita et al. - Beta Forging of Ti-6Al-4V: microstructure evolution and mechanical properties- Key Engineering Materials Vols. 554-557 (2013) pp 359-371 (C) (2013) Trans Tech Publications, Switzerland.

[10] D. Suárez Fernándeza, B. P. Wynnea, P. Crawforthc, K. Foxd, M. Jacksona -The effect of forging texture and machining parameters on the fatigue performance of titanium alloy disc components- 16 May 2020.

[11] Ali Mamedov et al. - Investigation of Mechanical and Microstructural Properties of Ti 6Al-4V Alloy Depending on Hot Forging Process Parameters - International Conference Technology of Plasticity, ICTP 2017.

[12] K. Y. Han and R. B. Thompson- Relationship between the two-point correlation of elastic constants and backscattered ultrasonic noise in two-phase- Iowa State University Ames, Iowa 50011- Thompson and D. E. Chimenti, Plenum Press, New York, 1995.

[13] Linxiao Yu- Understanding and improving ultrasonic inspection of jet-engine titanium alloy -Iowa State University 2004.

[14] Anton Van Pamel- Ultrasonic inspection of highly scattering materials-Imperial College London, Department of Mechanical Engineering-2015.

[15] Margetan, F. J., Umbach, J., Roberts- Inspection Development for Titanium Forgings- DOT/FAA/AR-05/46 - May 2007. 